Check for updates

Cite this: RSC Adv., 2019, 9, 36082

Received 31st May 2019

Accepted 18th October 2019

DOI: $10.1039 / c 9 r a 04118 d$

rsc.li/rsc-advances

\section{CSSF-CLIP-HSQMBC: measurement of heteronuclear coupling constants in severely crowded spectral regions $\dagger$}

\begin{abstract}
Aitor Moreno, ${ }^{a}$ Kine $\varnothing$ stnes Hansen ${ }^{\mathrm{b}}$ and Johan Isaksson (D) *c
A new pulse program development, a chemical shift selective filtration clean in-phase HSQMBC (CSSFCLIP-HSQMBC), is presented for the user-friendly measurement of long-range heteronuclear coupling constants in severely crowded spectral regions. The introduction of the chemical shift selective filter makes the experiment extremely efficient at resolving overlapped multiplets and produces a clean selective CLIP-HSQMBC spectrum, in which the desired coupling constants can easily be measured as an extra proton-carbon splitting in $\mathrm{f} 2$. The pulse sequence is also provided as a real-time homonuclear decoupled version in which the heteronuclear coupling constant can be directly measured as the peak splitting in f2. The same principle is readily applicable to IPAP and AP versions of the same sequence as well as the optional TOCSY transfer, or in principle to any other selective heteronuclear experiment that relies on a clean ${ }^{1} \mathrm{H}$ multiplet.
\end{abstract}

\section{Introduction}

Long-range heteronuclear scalar couplings contain important information about molecular relative configuration, structure identity and structural conformation. ${ }^{\mathbf{1 - 4}}$ The size of these couplings are in the same range as proton-proton scalar couplings, $0-15 \mathrm{~Hz}$, but are generally more complicated to measure accurately. There is a large number of different pulse sequences available for the measurement of long range heteronuclear scalar couplings, mainly divided into TOCSY basedand HMBC/HSQMBC-based methods. The pros and cons with these have been comprehensively reviewed. ${ }^{5-7}$ In short, no technique has so far proven to be a generally applicable method to measure long range couplings, but it is always a matter of choosing the most appropriate technique with regards to the amount of sample, the sample complexity (overlap), whether the carbon is protonated or not, and how many couplings need to be measured simultaneously.

A user-friendly approach for measuring long range protoncarbon scalar couplings has been proposed by Saurí et al. ${ }^{8}$ The CLIP-HSQMBC uses a selective pulse on a proton signal to remove interfering homonuclear couplings. This is crucial to assure pure absorptive lineshapes to allow the accurate measurement of coupling constants. The HSQMBC then

${ }^{a}$ Bruker BioSpin AG, Application Science Department, CH-8117 Fällanden, Switzerland ${ }^{b}$ Marbio, UiT - The Arctic University of Norway, Breivika, NO-9037 Tromsø, Norway ${ }^{c}$ Department of Chemistry, UiT - The Arctic University of Norway, Breivika, NO-9037 Tromsø, Norway.E-mail: johan.isaksson@uit.no

$\dagger$ Electronic supplementary information (ESI) available. See DOI: 10.1039/c9ra04118d produces carbon coupled in-phase multiplets in which the relevant proton-carbon coupling produces an extra splitting in f2 compared to the corresponding proton multiplet in an ordinary proton $1 \mathrm{D}$ spectrum.

Severely crowded spectral regions present a challenge for the measurement of coupling constants in general. Spectral overlap in both the proton and carbon dimensions of non-homonuclear coupled protons is not uncommon in complex molecules e.g. for (a) multiple residues of the same amino acid in modified peptides, (b) (deoxy)-ribose moieties in nucleic acids, (c) carbohydrates, (d) pseudo-symmetric parts of small molecules (see securidine A example below), or (e) in stretches of repeated atoms like for example in molecules containing (partly unsaturated) lipids. Another case is coincidentally overlapping proton signals coupling to the same carbon through long-range scalar coupling. This can arise for example in natural products containing many aliphatic protons like modified cyclic peptides, polyketides, macrolides, steroids, saponins, terpenoids, glycosides etc. Difficulties with spectral overlap can sometimes be circumvented by coupling the experiment to a TOCSY element, which situationally allows the selection of one out of several overlapping resonances. ${ }^{8}$ A 3D HSQCHSQMBC approach has also been proposed to address the problem of spectral overlap, ${ }^{9}$ as well as a $J$-scaled CLIPHSQMBC. ${ }^{10}$

In order to overcome this limitation we here propose to apply gradient enhanced proton chemical shift selective filtration $(\mathrm{CSSF})^{11}$ as the selection element in the CLIP-HSQMBC method. The ability to very cleanly select an unresolved multiplet in the proton dimension also results in a reduced number of observed correlations in the carbon dimension, thereby reducing the risk 
of inconvenient overlaps, both direct and folded/aliased, within the sampled carbon spectral width. We have denoted the pulse sequence development: CSSF-CLIP-HSQMBC.

An important limitation to get straightforward coupling measurements directly from in-phase separated peaks in the parent CLIP-HSQMBC is the requirement that no other proton coupled with the proton of interest may be excited by the selective pulse, as this will add dispersive contributions to the lineshape. It should be noted that this limitation is not overcome by the chemical shift selective filter even though it visually appears entirely clean. It is only through the selectivity of the shaped pulse that contributions from $J_{\mathrm{HH}}$ couplings can be avoided, whereas the CSSF cleans up any off-resonance chemical shifts that are excited by the selective pulse.

It is highly attractive to simplify the multiplet pattern of the crosspeaks, which results from the homo- and heteronuclear couplings, to a simple doublet in $\mathrm{f} 2$ split by the heteronuclear coupling constant. In the case where there is no spectral overlap, a PSYCHE version of HSQMBC to achieve spectrum-wide homonuclear decoupling has been reported. ${ }^{12}$ In order to achieve homodecoupling in the CSSF-CLIP-HSQMBC experiment, a version with real-time band-selective homodecoupling (bshd) during acquisition has been prepared. ${ }^{\mathbf{1 3 , 1 4}}$ We demonstrate that even though coupling constant measurement in the direct dimension of homodecoupled spectra can be treacherous, the heteronuclear coupling constants can be reliably measured directly as the splitting of the doublet in f2. This is possible as long as certain experiment conditions were scaling occurs are avoided.

\section{Results and discussion}

The new pulse sequence development, the CSSF-CLIPHSQMBC, makes use of a chemical shift selective filter as the means to achieve a clean in-phase selection of the proton of interest. ${ }^{11}$ CSSF is an iterative method that adds up the onresonance signal while off-resonance contributions are eliminated by destructive averaging because of differences in chemical shift evolution. The co-addition of FIDs makes this method extremely selective and allows the measurement of scalar couplings that may otherwise have been considered unmeasurable because of severe spectral overlap. Successful selection only requires a spectral separation of $1-2 \mathrm{~Hz}$ in the proton resonance frequency, and is thus able to resolve multiplets that appear to coincide.

The CSSF-CLIP-HSQMBC sequence shown in Fig. 1 contains two modules: the gradient-selected (gs)-CSSF ${ }^{11}$ and a CLIPHSQMBC $^{8}$ sequence, followed by ${ }^{1} \mathrm{H}$ detection in the absence of ${ }^{13} \mathrm{C}$ decoupling. The first part of the pulse sequence is the gsCSS filter, which allows the highly selective excitation of overlapping proton signals of interest with a reported resolution of up to $1.4 \mathrm{~Hz}$. The selectivity of the CSSF is given by $\Delta \nu=0.5 / t_{\max }$, where $t_{\max }$ is the maximum chemical shift evolution interval and $\Delta \nu$ is the chemical shift difference between the overlapping protons. The second part is a CLIP-HSQMBC sequence that allows the clean observation of ${ }^{13} \mathrm{C}$-isotopomer signals in which both the heteronuclear and homonuclear couplings exhibit

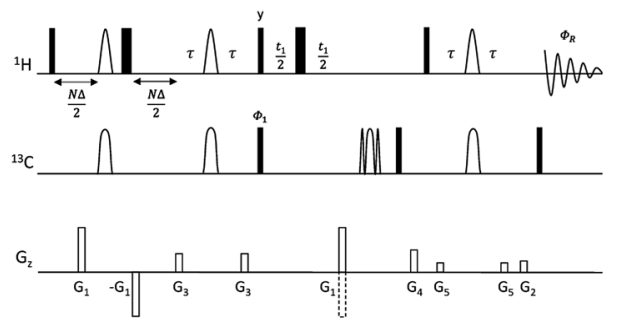

(a)

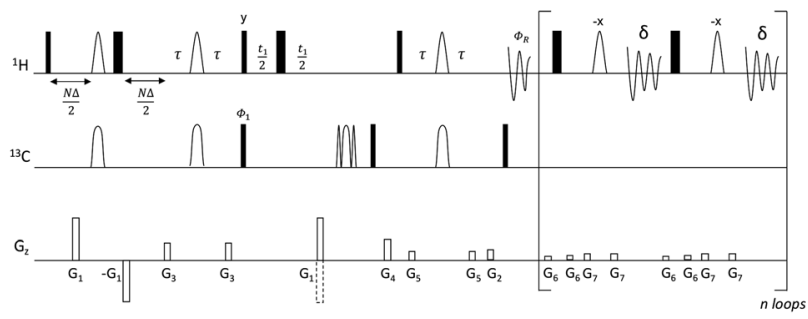

(b)

Fig. 1 Pulse sequences for the CSSF-CLIP-HSQMBC (a) and CSSFCLIP-HSQMBC_bshd (b) experiments. Narrow and wide rectangles represent $90^{\circ}$ and $180^{\circ}$ pulses, respectively, applied from the $x$ axis unless otherwise specified. Unfilled arcs on ${ }^{1} \mathrm{H}$ represent $180^{\circ}$ selective refocusing pulses, while unfilled arcs on ${ }^{13} \mathrm{C}$ represent $180^{\circ}$ adiabatic inversion and refocusing pulses, respectively. $D$ is the increment of the CSSF, $N=0,1,2 \ldots n$, where $t_{\max }=(n+1) D$. The delay $\tau^{\prime}\left(=1 /\left(4^{n} J_{\mathrm{CH}}\right)=\tau\right.$ $+p 180 / 2$, where $p 180$ is the duration of the selective $180^{\circ}{ }^{1} \mathrm{H}$ pulse) is an INEPT transfer delay. A minimum two-step phase cycle is applied: $\Phi_{1}=\Phi_{\mathrm{R}}=x,-x$. Gradients $G_{1}$ and $G_{2}$ are used for coherence selection using the echo-antiecho protocol, $G_{4}$ acts as a zz-filter, and $G_{1}, G_{3}, G_{5}$, $G_{6}$ and $G_{7}$ flank the selective refocusing proton pulses and hard $180^{\circ}$ pulses, respectively. The following pulsed field gradients were used: $G_{1}$ $=40 \mathrm{G} \mathrm{cm}^{-1}, G_{2}=10 \mathrm{G} \mathrm{cm}^{-1}, G_{3}=17 \mathrm{G} \mathrm{cm}^{-1}, G_{4}=25 \mathrm{G} \mathrm{cm}^{-1}, G_{5}=9$ $\mathrm{G} \mathrm{cm}^{-1}, G_{6}=1.5 \mathrm{G} \mathrm{cm}^{-1}, G_{7}=2.5 \mathrm{G} \mathrm{cm}^{-1}$. For the CSSF-CLIPHSQMBC_bshd experiment (b) homonuclear decoupling during the acquisition time $(A Q)$ is performed using refocusing blocks including a pair of hard and selective $180^{\circ} 1 \mathrm{H}$ pulses applied at intervals of $2 \delta=$ $\mathrm{AQ} / n$, where $n$ is the number of loops.

pure in-phase character. This is achieved by the selective $180^{\circ}$ proton pulses which eliminate homonuclear coupling modulations and hence avoid signal distortions. Further, heteronuclear antiphase components are eliminated by the application of $90^{\circ}$ carbon pulse before acquisition resulting in pure absorptive lineshapes. In order to speed up the acquisition, non-uniform sampling can readily be applied in the indirect dimension. The full pulse sequence is attached in the ESI. $\dagger$

\section{Quinine}

As a proof of principle, the CSSF-CLIP-HSQMBC was compared to the parent CLIP-HSQMBC experiment using a sample of $50 \mathrm{mM}$ quinine in DMSO- $d_{6}$ (Fig. 2). Even though the $\mathrm{H}^{\prime}{ }^{\prime}$ and $\mathrm{H}^{\prime}$ protons are only separated by $1.8 \mathrm{~Hz}$ in a proton $1 \mathrm{D}$ spectrum acquired at $400 \mathrm{MHz}$ proton frequency, the CSSF allows the selection of the near perfectly clean individual doublets using eight added FIDs $\left(t_{\mathrm{d} 0}=8\right)$ with different chemical shift evolution periods, effectively removing all off-resonance contributions (Fig. 2c and d). The clean doublets display the 


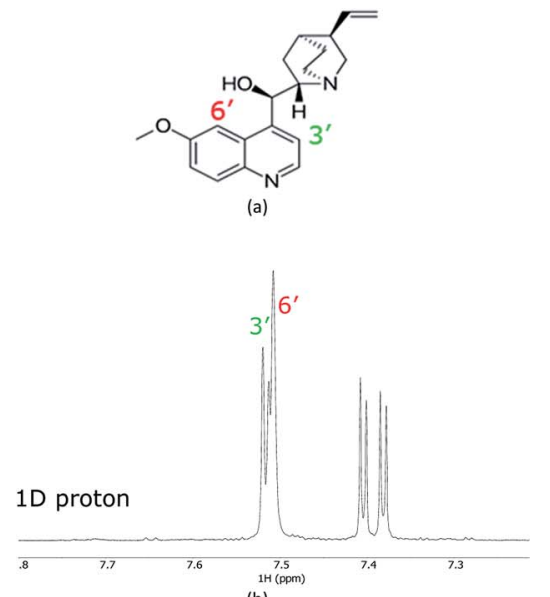

(b)

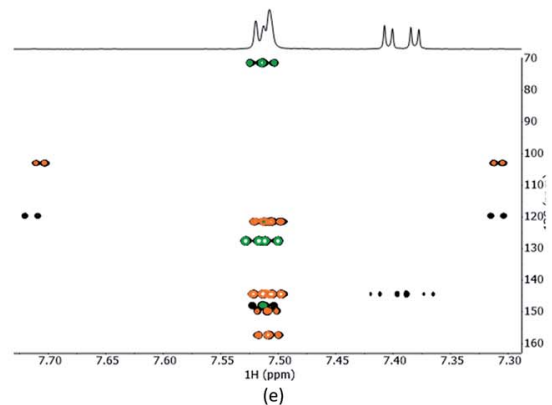

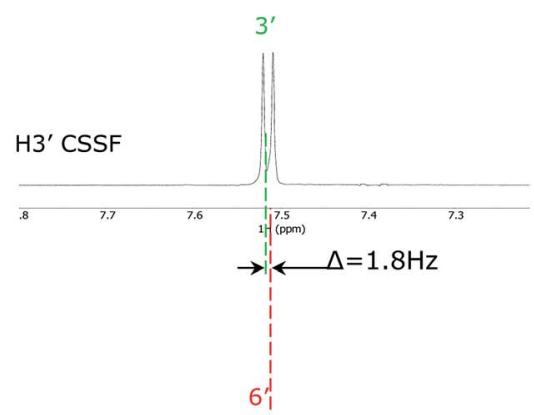
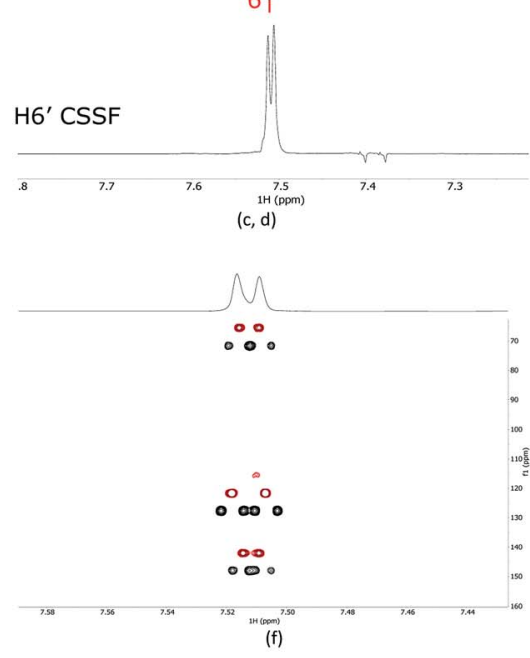

Fig. 2 (a) The structure of quinine, (b) 1D proton spectrum showing the partially overlapping H3' and H6' doublets, (c) the CSSF selected (selcssfzg) clean doublets of $\mathrm{H3}^{\prime}$ and (d) H6'. (e) Superimposed selective CLIP-HSQMBC (black), with CSSF-CLIP-HSQMBC of H3' (green) and H6' (orange) using the corresponding CSSF setting applied in (c) and (d). In (f) the H3' selected CSSF-CLIP-HSQMBC (black) is superimposed with an offset on the real-time bshd CSSF-CLIP-HSQMBC (red).

couplings ${ }^{3} J_{\mathrm{H}^{\prime} \mathrm{H}^{\prime}}=4.5 \mathrm{~Hz}$ and ${ }^{4} J_{\mathrm{H}^{\prime} \mathrm{H} 8^{\prime}}=2.8 \mathrm{~Hz}$. The corresponding CSSF-CLIP-HSQMBC produces clean 2D spectra consisting only of correlations originating at the selected proton and the observed splitting patterns are in this case identical to the original CLIP-HSQMBC sequence (Fig. 2e) as none of the carbons simultaneously couple strongly enough with both of the two overlapping protons ( $\mathrm{H}^{\prime}$ and $\mathrm{H6}^{\prime}$ ).

The multiple-bonds proton-carbon couplings can easily be measured as an extra splitting in the respective HSQMBC crosspeak. Both reference peaks are doublets and the CLIPHSQMBC peaks are resolved doublets of doublets.

The splittings due to homonuclear couplings in $\mathrm{f} 2$ in the CSSF-CLIP-HSQMBC spectrum can be eliminated by applying a real-time band-selective homonuclear decoupling scheme during acquisition (pulse sequence in Fig. 1b, and spectra in Fig. 2f). This is not as uncomplicated as it might first appear as pulsing during windowed acquisition is known to be able to cause $J$-scaling, phase shifts and chemical shift shifts. ${ }^{15-19}$ We do however show empirically that, as long as the length of the acquisition blocks are approximately twice as long as the selective pulse, no detectable scaling occurs and the method is robust for measuring long-range heteronuclear couplings in the direct dimension, under real-time band-selective homonuclear decoupling conditions (Fig. S1-S4 in the ESI $\dagger$ ).

\section{Securidine A}

The recently characterized natural product securidine A contains a spin system that has a high degree of chemical "symmetric equivalence" of positions 11-14 in an arginine sidechain spin-system, and thus results in nearly overlapped resonances that are very challenging to access (Fig. 3a). ${ }^{20}$ In this spin system, $\mathrm{H} 11$ and $\mathrm{H} 14$ are partially overlapping in the proton dimension $(\Delta \delta=6 \mathrm{~Hz}$, multiplet total width $=18.1 \mathrm{~Hz})$, and both protons have long range $\mathrm{CH}$ couplings to the same carbon atoms, C12 and C13. The attached protons, H12 and $\mathrm{H} 13$, are completely overlapping in the proton dimension $(\Delta \delta<$ $1 \mathrm{~Hz}$ ). This spin system was used to challenge the CSSF-CLIPHSQMBC sequence to evaluate the possibility of measuring the proton-carbon couplings individually in a near perfectly overlapped spin system. The CSSF could successfully select clean $6.2 \mathrm{~Hz}$ quartets from the partially overlapping H11 and H14 signals (Fig. $3 \mathrm{~b}$ ).

The selection profile was used in a CSSF-CLIP-HSQMBC to produce the individual $\mathrm{H} 11$ to $\mathrm{C} 12 / \mathrm{C} 13$ and $\mathrm{H} 14$ to $\mathrm{C} 12 / \mathrm{C} 13$ correlations. In this example the experiment was acquired with very high resolution in F1 without any interference from aliased peaks. These CLIP-HSQMBC resonances would be indistinguishable in an ordinary selective CLIP-HSQMBC (Fig. 3c). 


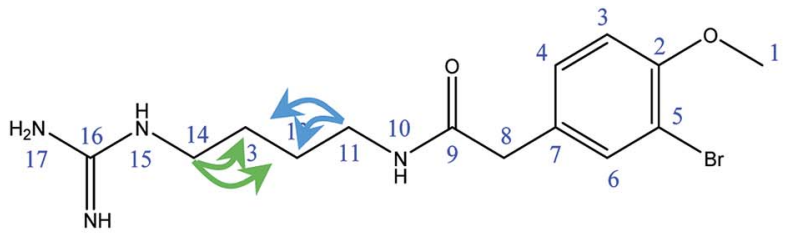

(a)

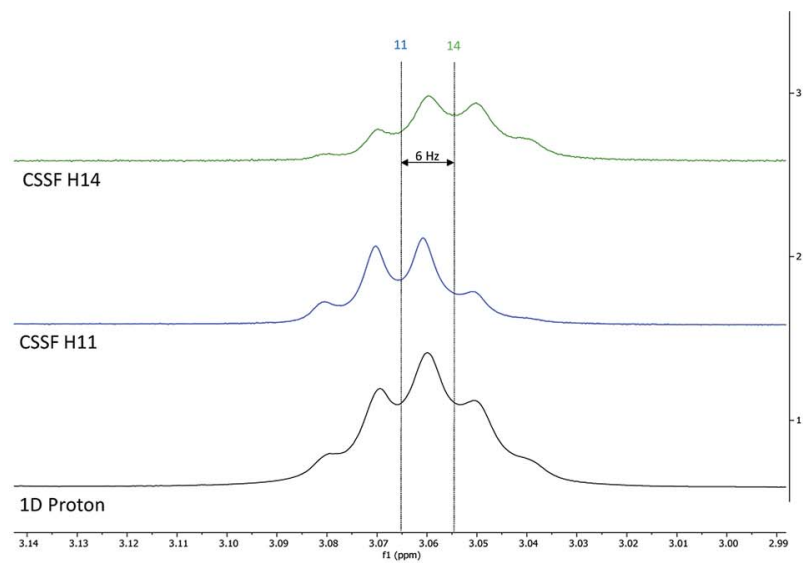

(b)

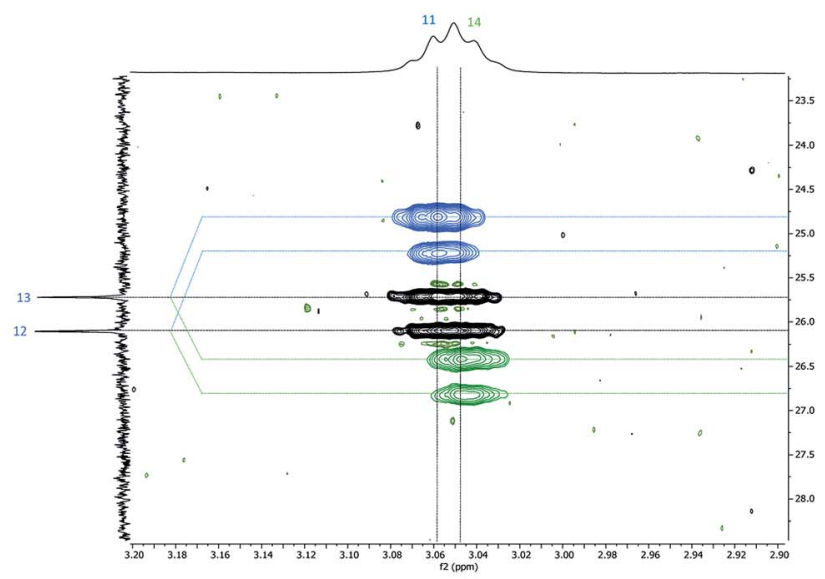

(c)

Fig. 3 (a) The chemical structure of securidine A, (b) stacked plots of the 1D proton spectrum of the overlapped $\mathrm{H} 11$ and $\mathrm{H} 14$ multiplets (bottom), with the cleanly filtered $\mathrm{H} 11$ (middle) and $\mathrm{H} 14$ (top) quartets. (c) The CSSF-CLIP-HSQMBC crosspeaks of the ${ }^{2} J_{\mathrm{H} 11 \mathrm{C} 12}$ and ${ }^{3} J_{\mathrm{H} 11 \mathrm{C} 13}$ (blue, offset above) as well as the ${ }^{2} J_{\mathrm{H} 14 \mathrm{C} 13}$ and ${ }^{3} J_{\mathrm{H} 14 \mathrm{C} 12}$ (green, offset below) with the parent CLIP-HSQMBC of the unresolved H11/H14 crosspeaks.

1D cross-sections through the CSSF-CLIP-HSQMBC peaks were extracted (Fig. 4c-f) and compared to the CSSF 1D reference peaks (Fig. 4a and b). The resulting multiplets were initially too complex to allow direct measurement of the extra $\mathrm{CH}$ splitting. The long range ${ }^{2} J_{\mathrm{C} 12 \mathrm{H} 11},{ }^{3} J_{\mathrm{C} 13 \mathrm{H} 11},{ }^{2} J_{\mathrm{C} 13 \mathrm{H} 14}$ and ${ }^{3} J_{\mathrm{C} 12 \mathrm{H} 14}$ coupling constants were therefore measured using two different approaches, both previously described. ${ }^{5}$

The first method uses the sum of couplings, measuring the separation of the two outermost maxima of the multiplets. The reference peak holds the sum of all proton couplings whereas the HSQMBC cross section holds the sum of all proton couplings plus the selected ${ }^{n} J_{\mathrm{CH}}$ coupling. The difference between the two sums yields the heteronuclear coupling constant.

The second method fits two HSQMBC cross sections to the reference peak by offsetting them (Fig. 4c-f), and the offset corresponds to the heteronuclear coupling constant. This method is preferred as it can be difficult to determine the outermost maximums of a multiplet if the signal to noise is low. Furthermore, fitting two multiplets by offsetting them is more robust in the presence of small phase distortions from weak $J_{\mathrm{HH}}$ coupling or imperfect purging, or in the presence of marginal second order effects.

The long-range heteronuclear proton carbon coupling constants in securidine A were thus successfully determined as follows: ${ }^{2} J_{\mathrm{C} 12 \mathrm{H} 11}=3.6,{ }^{3} J_{\mathrm{C} 13 \mathrm{H} 11}=4.6,{ }^{2} J_{\mathrm{C} 13 \mathrm{H} 14}=4.3$ and ${ }^{3} J_{\mathrm{C} 12 \mathrm{H} 14}=3.1 \mathrm{~Hz}$.

\section{Experimentals}

\section{Quinine}

NMR experiments were recorded on a $50 \mathrm{mM}$ sample of quinine in DMSO- $d_{6}$. The instrument was a Bruker Avance Neo Nanobay spectrometer operating at $400 \mathrm{MHz}$ for protons, equipped with a liquid nitrogen cooled broad-band observe cryoprobe (Prodigy $\mathrm{BBO})$ with cryogenic enhancement for ${ }^{1} \mathrm{H},{ }^{2} \mathrm{H}$ and all tunable $\mathrm{X}$ nuclei $\left({ }^{15} \mathrm{~N}-{ }^{31} \mathrm{P}\right)$. Experimental parameters of the CSSF-CLIPHSQMBC experiment (see Fig. 1): the length of the CSSF increment, $\Delta$, was $12.5 \mathrm{~ms}$ and a $15.8 \mathrm{~ms} 180^{\circ}$ Gaussian pulse was used as a selective $180^{\circ}$ pulse on protons. Adiabatic CHIRP shapes with a sweep width of $60 \mathrm{kHz}$ were used for inversion ( $0.5 \mathrm{~ms}$ ) and refocusing $(2 \mathrm{~ms}) 180^{\circ}$ carbon pulses. Smoothed square-shaped gradients of $1 \mathrm{~ms}$ duration were used, followed by a recovery delay of $200 \mu \mathrm{s}$. The gradient amplitude ratios for $G_{1}: G_{2}: G_{3}: G_{4}: G_{5} \quad$ are $\quad 80: 20.1: 33: 50: 17$. Gradient strengths are given as percentages of the absolute gradient strength of approximately $53.5 \mathrm{G} \mathrm{cm}^{-1}$. The acquisition times $t_{2}$ and $t_{1}$ were $0.78 \mathrm{~s}$ (spectral width $5263 \mathrm{~Hz}, 8 \mathrm{k}$ complex data points) and $6.36 \mathrm{~ms}$ (spectral width $10060 \mathrm{~Hz}, 128$ real data points), respectively. The relaxation delay was $1.5 \mathrm{~s}$ and 2 scans were accumulated for each of the CSSF increments $(n=8)$, resulting in 16 scans per $t_{1}$ increment. Zero filling to 512 points in F1, 8096 points in F2 and sine-squared window function in both F1 and F2 dimensions were applied before Fourier transformation of $2 \mathrm{D}$ data.

\section{Securidine A}

All NMR experiments were acquired on a sample of $2.0 \mathrm{mg}$ isolated securidine A dissolved in $1: 1$ DMSO- $d_{6}$ : chloroform- $d_{1}$. The instrument was a Bruker Avance III HD spectrometer operating at $600 \mathrm{MHz}$ for protons, equipped with an inverse detected TCI Helium Cryoprobe with cryogenic enhancement for ${ }^{1} \mathrm{H},{ }^{2} \mathrm{H}$ and ${ }^{13} \mathrm{C}$. The experimental parameters of the CSSF-CLIP-HSQMBC were typically: $\mathrm{ns}=8$, $t_{\mathrm{d} 0}=8, t_{\mathrm{d} 2}=2 \mathrm{k}, t_{\mathrm{d} 1}=64$. A $41.6 \mathrm{~Hz}$ wide Gaussian pulse was used for selective refocussing of the measured proton and the maximum gradient strength was $65.7 \mathrm{G} \mathrm{cm}^{-1}$, otherwise 


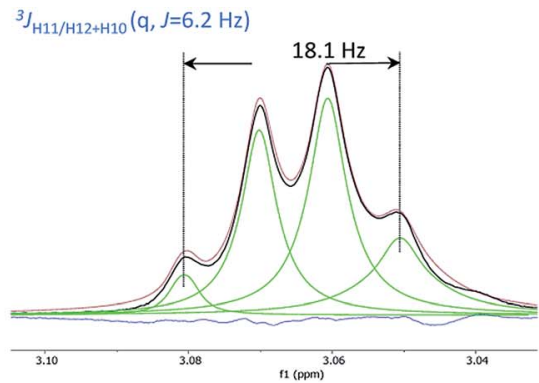

(a)

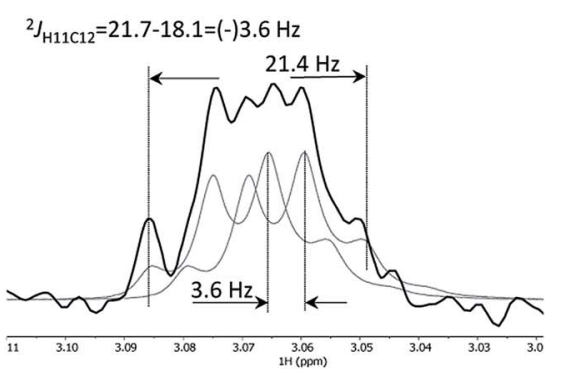

(c)

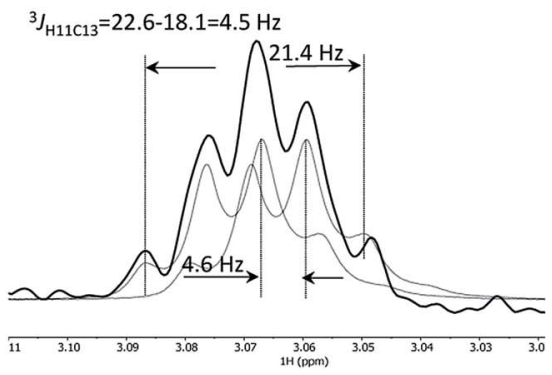

(e)

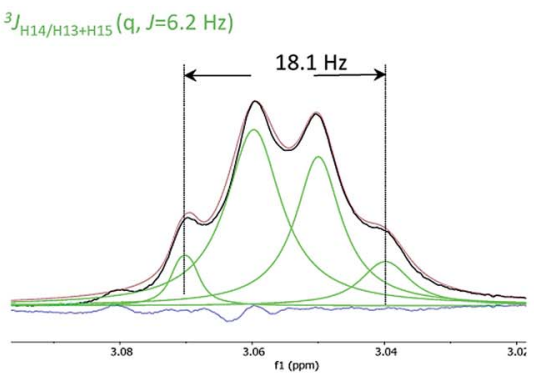

(b)

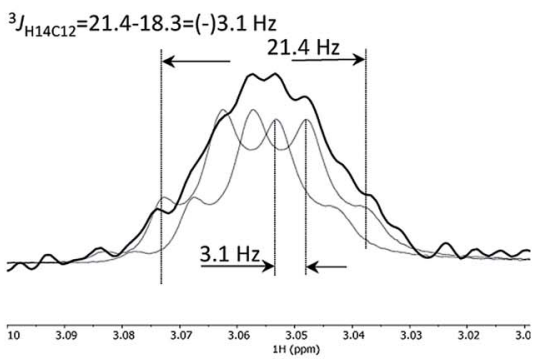

(d)

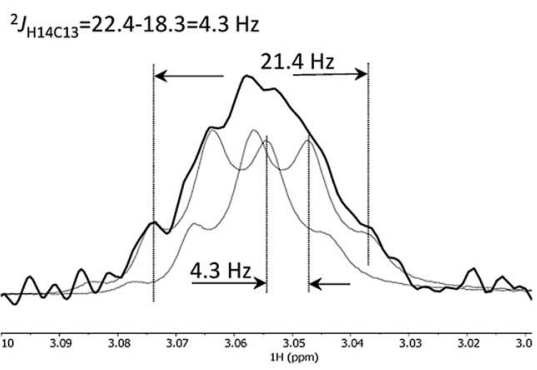

(f)

Fig. 4 Clean CSSF selected multiplets of $\mathrm{H} 11$ (a) and $\mathrm{H} 14$ of securidine $\mathrm{A}$, line fitted as quartets with $\mathrm{J}=6.2 \mathrm{~Hz}$ (b). Cross sections through the CSSF-CLIP-HSQMBC crosspeaks for H11/C12 (c), H14/C12 (d), H11/C13 (e) and H14/C13 (f). The CSSF multiplets were used as reference peaks, and two reference multiplets were offset to fit the CLIP-HSQMBC cross-section multiplet. This offset corresponds to the extra splitting caused by the ${ }^{n} J_{\mathrm{CH}}$ coupling.

identical settings were used as for quinine above. The data was zero filled to $8 \mathrm{k}$ complex points in the direct dimension and forward linear predicted to $1 \mathrm{k}$ complex points in the indirect dimension using 2 coefficients, and multiplied with a 45 degrees sine-squared function. The $1 \mathrm{D}$ cross section through each peak was then fitted to two reference peaks taken from the CSSF 1D, where the offset determined the ${ }^{n} J_{\mathrm{CH}}$ coupling constant.

\section{Conclusions}

In summary, we present a development that enables the measurement of long-range heteronuclear coupling constants in severely crowded spectral regions by using a chemical shift selective filter as means to eliminate any offresonance signals in the original CLIP-HSQMBC pulse sequence. We show that an offset of as little as $1-2 \mathrm{~Hz}$ is enough to allow for a clean filtration and accurate measurements of coupling constants. The CSSF selection is not dependent on being part of a spin system (like a selective TOCSY for instance), hence the method is generally applicable to any coupling of interest that is not perfectly isochronous with an interfering resonance, or are mutually coupled to each other. We further present a homonulcear decoupled version of the experiment and show that it is possible to reliably measure the heteronuclear coupling constants directly as the splitting in the direct dimension as long as the acquisition blocks are at least twice as long as the selective pulse used in the bshd element.

\section{Conflicts of interest}

There are no conflicts to declare. 


\section{Acknowledgements}

Generous funding from Digital Life Norway/Research Council of Norway, project ID: 269425, is gratefully acknowledged. The publication charges for this article have been funded by a grant from the publication fund of UiT The Arctic University of Norway.

\section{References}

1 P. E. Hansen, Carbon-hydrogen spin-spin coupling constants, Prog. Nucl. Magn. Reson. Spectrosc., 1981, 14(4), 175-295.

2 W. A. Thomas, Unravelling molecular structure and conformation-the modern role of coupling constants, Prog. Nucl. Magn. Reson. Spectrosc., 1997, 30(3), 183-207.

3 G. E. Martin, Qualitative and quantitative exploitation of heteronuclear coupling constants, in Annual Reports on NMR Spectroscopy, Academic Press, 2002, vol. 46, pp. 37-100.

4 N. Nath, Lokesh and N. Suryaprakash, Measurement and Applications of Long-Range Heteronuclear Scalar Couplings: Recent Experimental and Theoretical Developments, ChemPhysChem, 2012, 13(3), 645-660.

5 T. Parella and J. F. Espinosa, Long-range proton-carbon coupling constants: NMR methods and applications, Prog. Nucl. Magn. Reson. Spectrosc., 2013, 73, 17-55.

6 B. L. Marquez, W. H. Gerwick and R. Thomas Williamson, Survey of NMR experiments for the determination of $\mathrm{nJ}(\mathrm{C}, \mathrm{H})$ heteronuclear coupling constants in small molecules, Magn. Reson. Chem., 2001, 39(9), 499-530.

7 Y. Lin, Q. Zeng, L. Lin, Z. Chen and P. B. Barker, Highresolution methods for the measurement of scalar coupling constants, Prog. Nucl. Magn. Reson. Spectrosc., 2018, 109, 135-159.

8 J. Saurí, T. Parella and J. F. Espinosa, CLIP-HSQMBC: easy measurement of small proton-carbon coupling constants in organic molecules, Org. Biomol. Chem., 2013, 11(27), 4473-4478.

9 D. Uhrín, 3D HSQC-HSQMBC-increasing the resolution of long-range proton-carbon correlation experiments, J. Magn. Reson., 2002, 159(2), 145-150.
10 K. Buchberger, M. Walenta and K. Zangger, Extracting unresolved coupling constants from complex multiplets by a real-time J-upscaled SERF experiment, Magn. Reson. Chem., 2018, 56(10), 934-940.

11 P. T. Robinson, T. N. Pham and D. a. Uhrín, In phase selective excitation of overlapping multiplets by gradientenhanced chemical shift selective filters, J. Magn. Reson., 2004, 170(1), 97-103.

12 I. Timári, L. Szilagyi and K. Kövér, PSYCHE CPMGHSQMBC: An NMR Spectroscopic Method for Precise and Simple Measurement of Long-Range Heteronuclear Coupling Constants, Chem.-Eur. J., 2015, 21, 13939-13942.

13 L. Castañar, P. Nolis, A. Virgili and T. Parella, Full Sensitivity and Enhanced Resolution in Homodecoupled BandSelective NMR Experiments, Chem.-Eur. J., 2013, 19(51), 17283-17286.

14 J. Ying, J. Roche and A. Bax, Homonuclear decoupling for enhancing resolution and sensitivity in NOE and RDC measurements of peptides and proteins, J. Magn. Reson., 2014, 241, 97-102.

15 L. Emsley and G. Bodenhausen, Phase shifts induced by transient bloch-siegert effects in NMR, Chem. Phys. Lett., 1990, 168(3-4), 297-303.

16 N. F. Ramsey, Resonance Transitions Induced by Perturbations at Two or More Different Frequencies, Phys. Rev., 1955, 100(4), 1191-1194.

17 S. Stenholm, Saturation effects in RF spectroscopy. I. General theory, J. Phys. B: At. Mol. Phys., 1972, 5(4), 878-889.

$18 \mathrm{H}$. Matsuo, E. Kupce, H. Li and G. Wagner, Increased Sensitivity in HNCA and $\mathrm{HN}(\mathrm{CO}) \mathrm{CA}$ Experiments by Selective C $\beta$ Decoupling, J. Magn. Reson., Ser. B, 1996, 113(1), 91-96.

19 M. Sattler, J. Schleucher and C. Griesinger, Heteronuclear multidimensional NMR experiments for the structure determination of proteins in solution employing pulsed field gradients, Prog. Nucl. Magn. Reson. Spectrosc., 1999, 34(2), 93-158.

20 P. Michael, K. Ø. Hansen, J. Isaksson, J. H. Andersen and E. Hansen, A Novel Brominated Alkaloid Securidine A, Isolated from the Marine Bryozoan Securiflustra securifrons, Molecules, 2017, 22(7), 1236. 